\title{
Ad luxuriae instrumenta. Les objets dans leur contexte, entre la demeure et la tombe : le mobilier en ivoire et en os à Pompéi
}

Campagne 2020

\section{Anselme Cormier}

\section{(2) OpenEdition}

\section{Journals}

Édition électronique

URL : https://journals.openedition.org/baefe/2294

DOI : $10.4000 /$ baefe.2294

ISSN : 2732-687X

Éditeur

ResEFE

Référence électronique

Anselme Cormier, « Ad luxuriae instrumenta. Les objets dans leur contexte, entre la demeure et la tombe : le mobilier en ivoire et en os à Pompéi » [notice archéologique], Bulletin archéologique des Écoles françaises à l'étranger [En ligne], Italie, mis en ligne le 22 mai 2021, consulté le 24 mai 2021. URL : http://journals.openedition.org/baefe/2294 ; DOI : https://doi.org/10.4000/baefe.2294

Ce document a été généré automatiquement le 24 mai 2021.

\section{(c) (i) $\odot$}

Le Bulletin archéologique des Écoles françaises à l'étranger est mise à disposition selon les termes de la Licence Creative Commons Attribution - Pas d'Utilisation Commerciale - Pas de Modification 4.0 International. 


\section{Ad luxuriae instrumenta. Les objets dans leur contexte, entre la demeure et la tombe : le mobilier en ivoire et en os à Pompéi}

Campagne 2020

Anselme Cormier

\section{NOTE DE L'ÉDITEUR}

La présente chronique fait état de l'avancement d'un projet d'étude sur les ivoires et les os ouvragés découverts à Pompéi, qui s'inscrit dans l'axe de recherche du Centre Jean Bérard (USR 3133, CNRS-EFR) «Espaces, économie et artisanat dans les cités vésuviennes ", et le sous-axe "L'artisanat urbain et la vie économique antique à Pompéi ». Il est intégré à la Mission archéologique «Italie du Sud » (2020-2023), sous l'égide du ministère de l'Europe et des Affaires étrangères. Je remercie à cet égard Claude Pouzadoux et Priscilla Munzi, directrice et directrice-adjointe du Centre Jean Bérard.

\section{NOTE DE L'AUTEUR}

Date précise de l'opération : 14-25 janvier et 2-10 octobre 2020

Autorités nationales présentes : Parco Archeologico di Pompei, Museo Archeologico Nazionale di Napoli - MiC Composition de l'équipe de terrain : Anselme Cormier, Université catholique de l'Ouest, CHUS (Centre de recherche Humanités et Sociétés); Centre Jean Bérard (USR 3133, CNRS-EFR), archéologue. Letizia Nonne, archéologue, Université Libre de 
Bruxelles, Atelier de restauration, Fédération des archéologues de Wallonie et de Bruxelles, restauratrice d'objets archéologiques.

Partenariats institutionnels : Centre Jean Bérard (USR 3133, CNRS-EFR), Parco

Archeologico di Pompei, Museo Archeologico Nazionale di Napoli, Université libre de Bruxelles

Établissement éditeur : CJB

Établissements porteurs de l'opération : CJB

Remerciements : Je remercie le directeur du Museo Archeologico Nazionale di Napoli (MANN), Paolo Giulierini, mais aussi Marialucia Giacco, funzionario archeologo conservatore e Responsabile Ufficio Mostre Italia/Estero au sein de la direction scientifique, ainsi que Floriana Miele, funzionario archeologo conservatore e Responsabile Ufficio Catalogo, Andrea Milanese, directeur de l'Ufficio di Museologia e Documentazione storica, pour leur disponibilité et leur aide, ainsi que Maria Morisco, assistente alla fruizione, les consegnatari du musée et le photographe Giorgio Albano.

Je remercie enfin le directeur du Parco Archeologico di Pompei, Massimo Osanna, Stefania Giudice, funzionario restauratore, et Luana Toniolo, funzionario archeologo, pour les dépôts archéologiques et pour la Regio V; mais aussi Domenico Busiello et Ulderico Franco, les consegnatari responsables des dépôts, pour leur accueil et leur aide.

Données scientifiques produites :

https://centrejeanberard.cnrs.fr/spip.php?article405\&lang=fr

Chroniques de l'EFR :

https://journals.openedition.org/cefr/4367

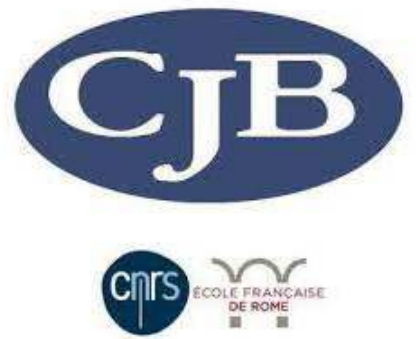

\section{Objet de l'étude}

1 Ce projet se propose de développer une recherche sur l'art et l'artisanat de l'ivoire et de l'os à l'époque romaine, essentiellement à Pompéi, comme lieu central, et d'éclairer cet aspect de la culture matérielle des sociétés italiennes aux $\mathrm{I}^{\mathrm{er}}$ siècle av. et $\mathrm{I}^{\mathrm{er}}$ siècle apr.J.-C., à partir de collections particulièrement fournies qui constituent un échantillonnage à l'échelle d'une ville entière, en dressant un état de la question sur les usages et la production liée à cet artisanat. Le matériel conservé au musée de Naples et dans les dépôts du site même (la Casa Bacco en particulier) est en cours d'étude depuis maintenant trois années, lors de missions ponctuelles effectuées sur place. Sur les quelques 2600 artefacts et fragments actuellement dénombrés, une classification d'environ 1400 d'entre eux a déjà été réalisée, selon leur matériau - ivoire ou os -, leurs usages, leurs formes, les lieux et contextes de découverte, quand ces informations sont 
disponibles. Chaque élément a ainsi été observé, identifié, inventorié, mesuré, photographié et précisément décrit.

2 Au musée de Naples, les collections n'ont fait jusqu'à présent l'objet que de peu de publications : en 1963, A. De Franciscis publiait quelques éléments marquants en ivoire et en os $^{1}$ et, en 1986, M. R. Borriello proposait un chapitre sur Gli avori et gli ossi lavorati ${ }^{2}$, dans lequel elle inventoriait et décrivait 26 pièces de valeurs particulièrement significatives. Par ailleurs, différents articles et monographies évoquaient des pièces éparses. Ainsi, en 2018, en accord avec Valeria Sampaolo, puis Marialucia Giacco, a été décidée la création d'un catalogue général des ivoires et des os ouvragés, dans l'optique d'une publication par les éditions du musée. Rappelons que les trois inventaires historiques généraux, points de départ de la recherche, ont été chacun successivement initiés au XIX siècle : 1826 pour l'Inventario Arditi, du Real Museo Borbonico ; 1852 pour l'Inventario Sangiorgio, toujours du Real Museo Borbonico (qui intègre le précédent) ; de 1863 à 1871 pour le troisième, l'Inventario Fiorelli, ou Inventario Generale Soprintendenza Antichità Campania. Ce dernier intègre également le précédent, et par conséquent le tout premier, ainsi que des données récentes. Mais l'intégralité des informations fournies par les deux premiers n'apparaît pas systématiquement dans l'inventaire général. Ainsi, pour les objets découverts dans les premiers temps des dégagements, certains ont parfois changé de numéro, quand d'autres les ont perdus. Afin de les compléter, de nouvelles numérotations ont par conséquent été proposées et incluent désormais des informations concernant les cassettes de conservation, à des fins de localisation plus aisée. Le travail s'effectue à l'aide des logiciels FileMaker Pro, Indesign et Excel, dans l'optique de croisements de données.

\section{Actions menées en 2020}

3 Au cours de l'année 2020, il n'a été possible de se rendre sur place qu'à deux reprises seulement, du 14 au 25 janvier et du 2 au 10 octobre. Ces deux missions ont vu l'intervention d'une restauratrice, Letizia Nonne', pour la mise en place d'un programme de consolidation et/ou de restauration de pièces particulièrement fragiles ou détériorées.

Consultation des archives, recoupements, localisations et précisions contextuelles ont ainsi pu être opérées, permettant de donner progressivement une visibilité archéologique plus grande, et de constituer des typologies autour de catégories définies: l'instrumentum et les outils, les éléments d'ameublement, les éléments de coffrets, le petit mobilier, les instruments de musique, la parure, les ustensiles de toilette, les éléments de jeu, les militaria. À l'époque romaine, l'ivoire était un matériau de luxe extrêmement prisé, et porteur d'une symbolique particulière. Son utilisation allait du petit instrument de la vie quotidienne à la décoration ou même la constitution de meubles ou d'objets votifs. Il en est de même pour l'os, matériau au coût infiniment moindre, mais capable d'imiter l'ivoire, voire de tenter de le remplacer. Il était utilisé à tous les degrés de l'échelle sociale, également pour la fabrication et la décoration d'objets, allant de la tabletterie au placage de meuble. Quantité d'ivoire et d'os a été retrouvée dans les fouilles archéologiques et, à ce titre, Pompéi constitue un exemple particulièrement caractéristique, mais aussi unique du fait des conditions de sa préservation, ouvrant ainsi des perspectives de compréhension de la culture matérielle 
liée à ses usages et à ses significations, tant dans les contextes domestiques que dans les contextes funéraires.

5 La première mission a été consacrée pour partie aux réserves de Pompéi et pour partie au musée de Naples. Dans les deux cas l'étude s'est concentrée sur l'inventaire et la construction de la base de données, mais aussi pour le musée sur la continuation de l'étude des archives historiques, permettant de poursuivre la constitution du catalogue des artefacts en ivoire et en os, augmentant ainsi l'étude des pièces déjà inventoriées. Une publication sur le mobilier du musée a d'ailleurs vu le jour au premier semestre 2020, dans la Chroniques des activités archéologiques de l'École française de Rome, traitant précisément d'éléments de lits mis au jour dans les demeures au cours des XVIII et XIX ${ }^{\mathrm{e}}$ siècles ${ }^{4}$.

\section{La maison I 16, 3}

6 L'étude des ivoires et de os conservés dans les dépôts du site de Pompéi s'est concentrée sur la maison I 16, 3. En 2011, Grete Stefani avait publié les résultats d'une enquête qu'elle avait menée, relative à des photographies des années 1950 montrant Amedeo Maiuri observant la mise au jour de corps. Or, trop peu d'indications existaient pour en déterminer le lieu exact de provenance. L'enquête avait cependant abouti à la maison I 16, 3, qui renfermait en outre une quantité importante d'objets manufacturés de valeur - des bijoux en particulier-, mais aussi de l'os ouvragé. La publication procède ainsi à une description de l'enquête, mais également à un inventaire du matériel ${ }^{5}$. Sur place cependant, après la restauration du lieu, avaient été laissés de nombreux éléments manufacturés non répertoriés dans l'inventaire, dont des fragments d'amphores ainsi qu'un crâne humain, mais aussi, aux dires de Grete Stefani, des os d'animaux qui portaient des traces d'outillage, auxquels l'auteure fait cette fois référence dans son article ${ }^{6}$. En 2019, un examen in situ a de fait révélé, en partie éparpillés et en partie entreposés dans des cassettes, d'une part des épiphyses sciées, et d'autre part les restes d'un squelette animal, qui s'est avéré être celui d'un âne, d'après l'analyse de Martine Leguilloux ${ }^{7}$, que je remercie pour son expertise. L'hypothèse de la présence d'un atelier du travail de l'os est désormais évoquée, nécessitant un approfondissement de la recherche en ce sens. En 2020, outre la continuation de l'élaboration de l'inventaire, a été initié un travail de consolidation de certaines pièces, en particulier provenant de la maison $\mathrm{I} 16,3$, et d'une réflexion préparatoire à la programmation à venir de l'étude archéologique du lieu, qui permettra d'en déterminer les caractéristiques.

7 En l'état actuel de son avancement, l'étude du matériel provenant de cette maison a mis en évidence la variété des typologies fonctionnelles des artefacts et fragments conservés dans le dépôt de la Casa Bacco, et d'autre part un lot de déchets de manufacture, actuellement entreposé dans l'une des pièces de la maison.

8 Au cours des fouilles de 1957, entre septembre et octobre surtout, le journal fait état d'une concentration de matériel et mobilier divers retrouvé dans la pièce immédiatement à gauche en entrant, donnant sur le vestibule; ils étaient manifestement entreposés dans une grande armoire dont avaient subsisté de nombreuses charnières en os et une serrure: objets précieux, bijoux, monnaies, bronzes, jetons en pâte de verre..., et plusieurs éléments en os et en ivoire. Dans leur article sur les objets liés au travail pictural à Pompéi ${ }^{8}$, Marie Tuffreau-Libre, Isabelle 
Brunie et Sébastien Daré émettent l'hypothèse que ce matériel avait probablement été rassemblé et entreposé dans cette armoire alors que la maison était en travaux au moment de l'éruption - comme d'ailleurs les maisons 2 et 4 de l'insula. Et cet état de fait expliquerait également la présence de traces de couleur sur un certain nombre d'objets en os, et surtout des spatules, pour certaines détournées de leur fonction première pour être utilisées par les peintres 9 . L'observation d'autres pièces évoquées ${ }^{10}$ tend à nous faire penser qu'il pourrait s'agir soit de déchets de manufacture, soit de pièces inachevées ${ }^{11}$.

9 Le nombre d'artefacts achevés et pour le moment répertoriés s'élève à 46 , dont 10 en ivoire et 36 en os (hors les éléments de la charnière de l'armoire), pour un total de 192 fragments ou pièces complètes. Huit catégories composent cet ensemble, qui peuvent être subdivisées en plusieurs types :

\begin{tabular}{|c|c|}
\hline \multicolumn{2}{|r|}{ Catégorie 1. L'instrumentum et les outils } \\
\hline- & $\begin{array}{l}\text { Un instrument de mesure en forme de lame (en os), interprété comme une règle plate, } \\
\text { finement graduée et divisée, avec une grille de perspective }{ }^{\mathrm{a}} \text {, mais interprétée plus récemment } \\
\text { comme une règle de réduction }^{\mathrm{b}} \text {; }\end{array}$ \\
\hline- & des ustensiles de filage : trois fuseaux en os ; \\
\hline- & une navette de tissage (?) en os ; \\
\hline - & $\begin{array}{l}\text { quatre lames à profil sinusoïdal en os, dont chaque face est en relief (la fonction n'est pas } \\
\text { déterminée). }\end{array}$ \\
\hline \multicolumn{2}{|r|}{ Catégorie 2. Les éléments d'ameublement } \\
\hline- & Un médaillon décoratif de meuble, en ivoire, avec tête de Ménade de profil ; \\
\hline- & une applique décorative rectangulaire en os et percée de trois trous ; \\
\hline- & nombreux charnons en os; \\
\hline- & un ensemble fragmentaire de montants de lit (os, fer, bois) (fig. 1). \\
\hline
\end{tabular}


Fig. 1. Fragment de montant de lit, os, bois et métal, de Pompéi I 16, 3, inv. 1200 I.

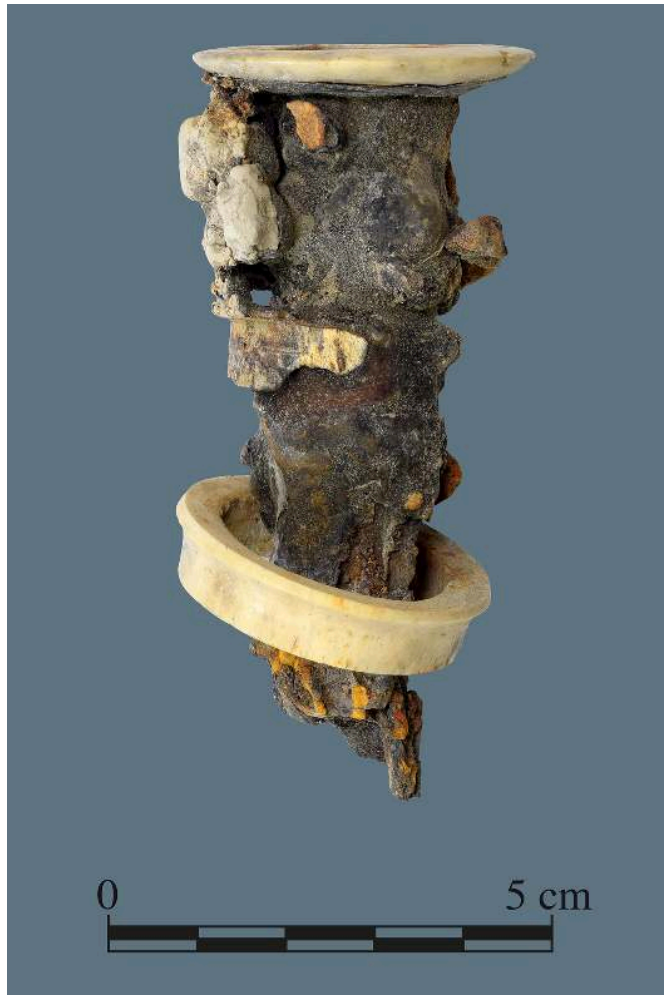

A. Cormier, sur concession du MiC - PA Pompei. Tous droits réservés.

\begin{tabular}{|l|l|l|}
\hline \multicolumn{2}{|l|}{ Catégorie 3. Les éléments de coffrets } \\
\hline- & $\begin{array}{l}\text { Neuf petits éléments figurés de placages décoratifs en os, à motifs de chapiteaux et de décors } \\
\text { géométriques, constitutifs de subtils ornements. }\end{array}$ \\
\hline & Catégorie 4. Le petit mobilier \\
\hline- & Un couvercle de pyxide en ivoire (fig. 2$) ;$ \\
\hline- & un couvercle de boîte rectangulaire à glissière en ivoire, incomplet; \\
\hline- & $\begin{array}{l}\text { une plaquette rectangulaire en ivoire, fragmentaire, probablement constitutive du fond de la } \\
\text { même boîte que le couvercle ; }\end{array}$ \\
\hline- & un aryballe en ivoire (fig. 3 ). \\
\hline
\end{tabular}


Fig. 2. Couvercle de pyxide, ivoire, de Pompéi I 16, 3, inv. 11909B.

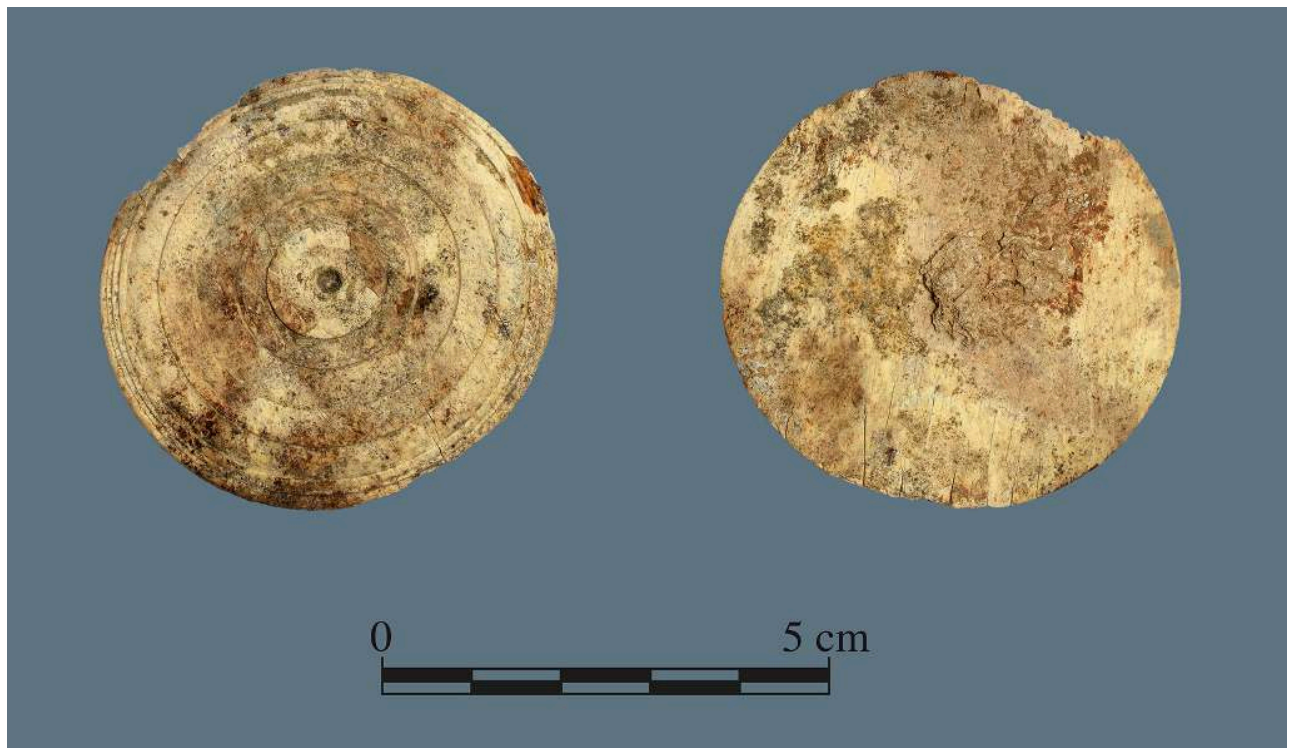

A. Cormier, sur concession du MiC - PA Pompei. Tous droits réservés.

Fig. 3. Aryballe, ivoire, de Pompéi I 16, 3, inv. 11879.

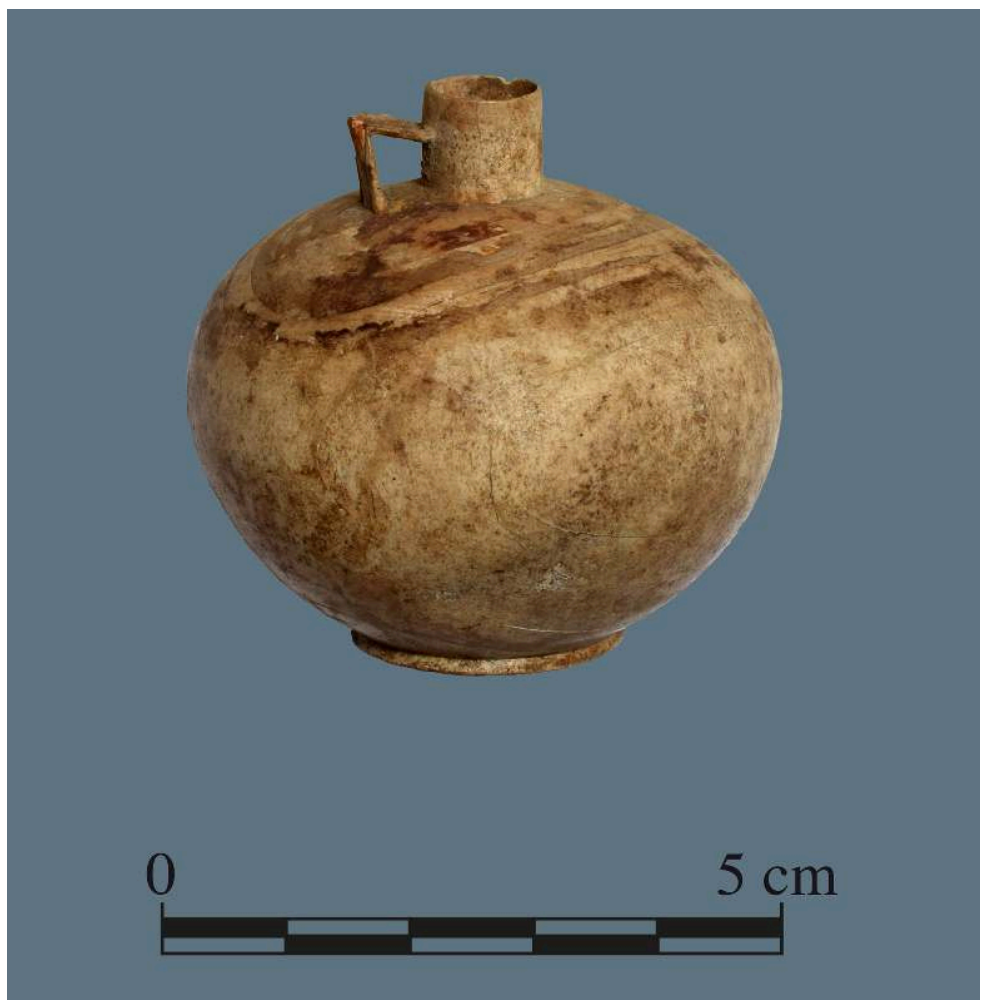

A. Cormier, sur concession du MiC - PA Pompei. Tous droits réservés

\begin{tabular}{|c|c|}
\hline & Catégorie 5 . La parure \\
\hline & - Un pendentif (?) en ivoire, bombé et orné de liserés radiaux; \\
\hline
\end{tabular}


un pendentif en os, de forme circulaire et orné d'un visage de profil incisé ;

Catégorie 6. Les ustensiles de toilette

Quatre spatules (?) plates en os, dont deux comportent une extrémité en forme de doigt, une autre dont le bord est en double liseré séparé par une gorge, la quatrième de forme trapézoïdale ;

un peigne en ivoire et deux peignes en os, fragmentaires.

Catégorie 7. Les éléments de jeu

Sept jetons en os ;

une flèche de jeu (?).

Catégorie 8. Indéterminé

Un élément discoïdal en ivoire ;

une plaquette ;

six lames fragmentaires, portant des traces de peinture.

10 À ces artefacts s'ajoutent des ébauches et des déchets de manufacture. Deux ensembles distincts sont ainsi à signaler. Le premier est un lot de baguettes, lames et lamelles dont une partie s'apparente à des déchets de manufacture et des ébauches (fig. 4). L'autre lot est constitué de rebuts d'artisanat (évoqué plus haut) : il s'agit d'épiphyses d'os, essentiellement de métatarses de bovidés, laissant voir sur plusieurs d'entre elles l'esquille d'arrachement qui a achevé le sciage transversal (fig. 5). Une étude ultérieure de ces deux ensembles permettra d'en proposer une analyse approfondie et d'apporter les précisions requises, qui seront avantageusement complétées par l'étude archéologique de la maison. 
Fig. 4. Baguettes, lames et lamelles s'apparentant à des déchets de manufactures et à des ébauches, os, de Pompéi I 16, 3, inv. 11938.

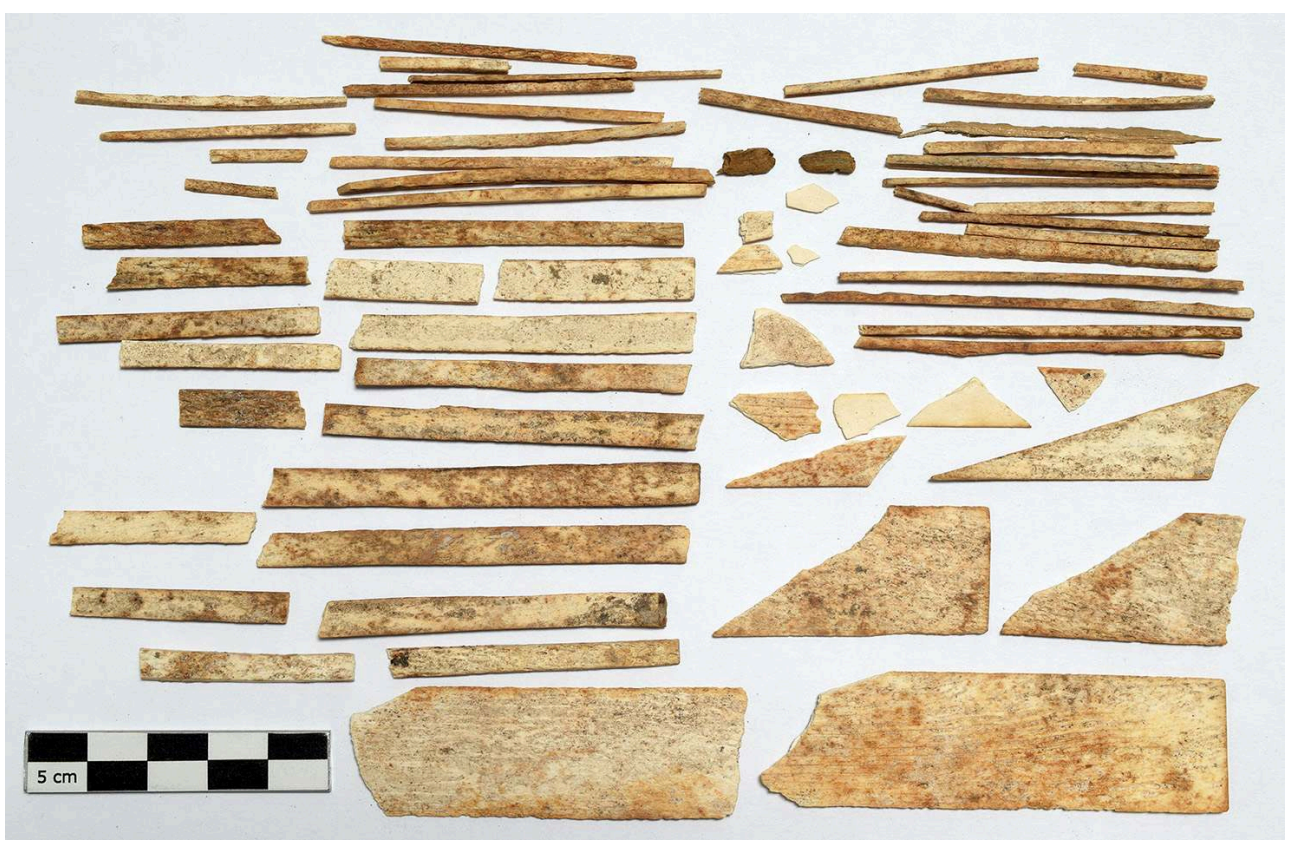

A. Cormier, sur concession du MiC - PA Pompei. Tous droits réservés.

Fig. 5. Déchets d'artisanat (épiphyses sciées), de Pompéi I 16, 3.
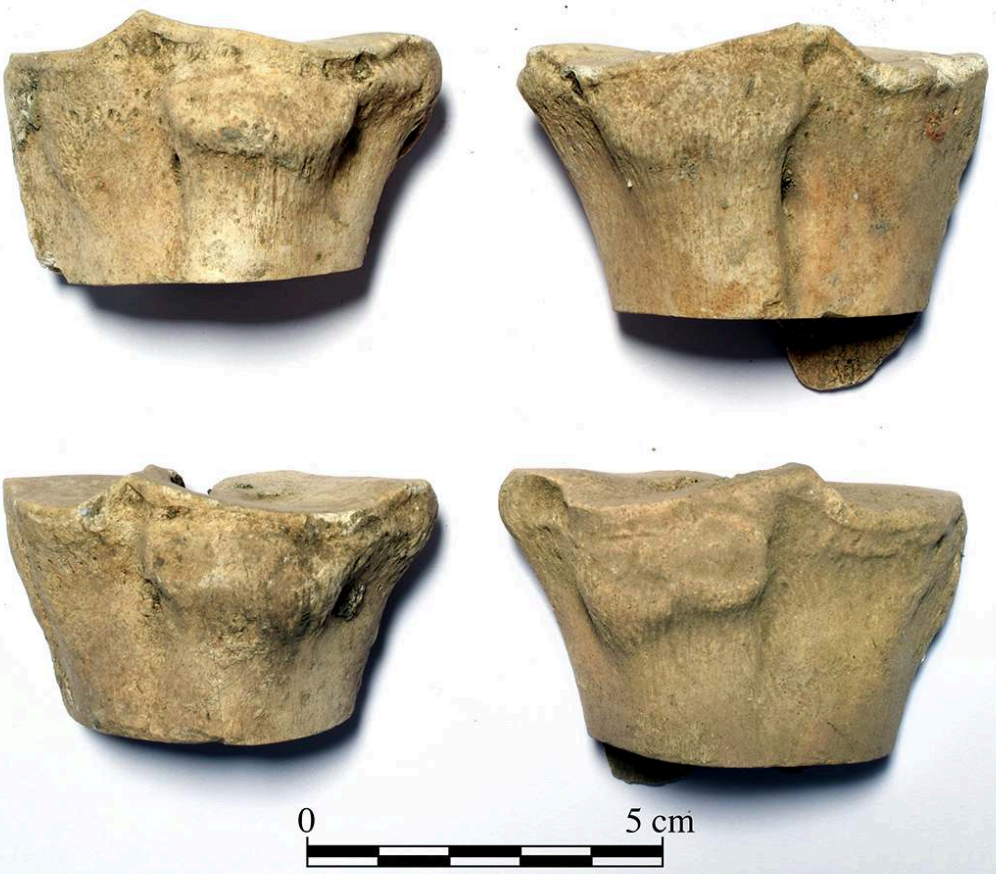

A. Cormier, sur concession du MiC - PA Pompei. Tous droits réservés. 


\section{La nécropole de la Porta Nocera}

11 La deuxième mission a été consacrée au site de Pompéi exclusivement : parallèlement à l'étude de la maison I 16, 3, l'attention s'est portée sur le champ funéraire, avec l'étude du mobilier en ivoire et en os mis au jour lors des fouilles de la nécropole de la Porta Nocera, au sein du programme de recherche de l'École française de Rome ${ }^{12}$ Pompéi Fouilles de la nécropole de Porta Nocera, campagne 2020, mené sous la direction de William Van Andringa et Henri Duday. Les dernières données recueillies ${ }^{13}$ permettent de commencer à envisager des ponts particulièrement intéressants entre les objets provenant des tombes et ceux trouvés dans les contextes domestiques: instrumentum (fuseaux et fusaïoles, quenouilles, manches...) ; effets féminins, parures et ustensiles de toilette; éléments décoratifs d'ameublement et de coffrets (appliques, placages, cylindres, anneaux, bandeaux). Signalons par exemple une typologie similaire constatée entre les fragments en ivoire d'un lit funéraire retrouvé dans une tombebûcher ${ }^{14}$, et certains exemplaires découverts dans les demeures ${ }^{15}$, et actuellement en cours d'étude.

\section{BIBLIOGRAPHIE}

CORMIER 2019

Anselme Cormier, «Les ivoires et les os ouvragés », in Rapport de fouilles 2019, W. Van Andringa, Th. Creissen et H. Duday, Naissance et développement d'un paysage funéraire romain (I ${ }^{\text {er }}$ siècle av. $I^{e r}$ siècle apr. J.-C.). Aux portes de la ville et le long de la route de Nocera, Pompéi. Fouille de la nécropole romaine de Porta Nocera, École française de Rome, École Pratique des Hautes Études (UMR 8546 CNRS/ENS AOrOc), Evéha International, Soprintendenza Archeologica di Pompei, p. 471-528.

CORMIER 2020a

Anselme Cormier, «Les lits en ivoire et les lits en os du Musée archéologique de Naples », CEFR, Les cités vésuviennes, 2020, en ligne, http://journals.openedition.org/cefr/4367, consulté le 30 janvier 2021.

CORMIER 2020b

Anselme Cormier, «Les ivoires et les os ouvragés de l'enclos 1F », Rapport de fouilles 2020, in William Van Andringa, Henri Duday (éd.), Pompéi. Fouille de la nécropole romaine de Porta Nocera, École française de Rome, avec la collaboration de l'École Pratique des Hautes Études (UMR 8546 CNRS/ENS AOrOc, Université PSL) et du Parco Archeologico di Pompei, p. 271-310.

DE FRANCISCIS 1963

Alfonso de Franciscis, « Avorio e osso », in Il Museo Nazionale di Napoli, Cava de' Tirreni, Di Mauro, 1963.

ERISTOV, PARZYSZ 2018

Hélène Eristov, Bernard Parzysz, « Une intrigante lame d'ivoire pompéienne », Rivista di Studi pompeiani XXIX, 2018, p. 21-29. 
STEFANI, BORGONGINO 2010

Grete Stefani, Michele Borgongino, « Note in margine ad un rinvenimento della Regio I di Pompei. La domus I 16, 3 e la sua documentazione di scavo », Rivista di Studi Pompeiani XXI, 2010, p. 87-99.

TUFFREAU-LIBRE, BRUNIE, DARÉ 2013

Marie Tuffreau-Libre, Isabelle Brunie, Sébastien Daré, « Peinture et perspective à Pompéi, un ensemble d'objets liés au travail pictural (I 16, 2.3.4) », Rivista di studi pompeiani XXIV, 2013, p. 53-69.

\section{NOTES}

1. DE FRANCISCIS 1963.

2. BORRIELLO 1986, p. 111-112 ; cat. p. 230-233.

3. Archéologue et restauratrice d'objets archéologiques, Université Libre de Bruxelles, Atelier de restauration (Fédération des archéologues de Wallonie et de Bruxelles).

4. CORMIER 2020a.

5. STEFANI, BORGONGINO 2010.

6. STEFANI, BORGONGINO 2010, p. 95.

7. Université Aix-Marseille, UMR 7299 CNRS-CCJ, Centre Archéologique du Var.

8. TUFFREAU-LIBRE, BRUNIE, DARÉ 2013.

9. TUFFREAU-LIBRE, BRUNIE, DARÉ 2013, p. 57-58.

10. TUFFREAU-LIBRE, BRUNIE, DARÉ 2013, p. 61, fig. 17, n. 4 à 7 et p. 62, fig. 18, n. 9 à 11.

11. Comme indiqué en infra, cet aspect sera approfondi au cours d'une étude à venir.

12. En collaboration avec l'École Pratique des Hautes Études (UMR 8546 CNRS/ENS AOrOc, Université PSL), et le Parco Archeologico di Pompei.

13. CORMIER $2020 \mathrm{~b}$.

14. CORMIER 2019.

15. CORMIER 2020a, pl. 2, n. 2 et 3 par ex. Voir également la paire de montants conservée dans les dépôts du site, inv. 1622a et 1622b, de I 6, 4 (en cours d'étude et de publication). 


\section{INDEX}

\section{Année de l'opération : 2020}

lieux https://ark.frantiq.fr/ark:/26678/pcrt7ya9wO0Xhb

chronologie https://ark.frantiq.fr/ark:/26678/pcrtZTmusVUU24

peuples https://ark.frantiq.fr/ark:/26678/pcrtkERiWybjc4

Thèmes : $\mathrm{CJB}$

sujets https://ark.frantiq.fr/ark:/26678/pcrtuNA5xRSr9l, https://ark.frantiq.fr/ark:/26678/ pcrtEaFZFvpInN, https://ark.frantiq.fr/ark:/26678/pcrtlflojXmhAi, https://ark.frantiq.fr/ark:/ 26678/crtiUEzUHzs1A, https://ark.frantiq.fr/ark:/26678/crtQMTce606Z7, https://ark.frantiq.fr/ ark:/26678/pcrtN1nPRZ3mUo, https://ark.frantiq.fr/ark:/26678/pcrtPfgeWSPnIt

\section{AUTEURS}

\section{ANSELME CORMIER}

Université catholique de l'Ouest, CHUS (Centre de recherche Humanités et Sociétés) ; Centre Jean Bérard (USR 3133, CNRS-EFR) 